\title{
Carrier recombination mechanism and photovoltage deficit in 1.7-eV band gap near-stoichiometric $\mathrm{Cu}(\mathrm{In}, \mathbf{G a}) \mathrm{S}_{2}$
}

\author{
Sudhanshu Shukla, ${ }^{*}$ Damilola Adeleye $\odot$, Mohit Sood $\odot$, Florian Ehre, Alberto Lomuscio $\odot$, Thomas Paul Weiss, \\ Daniel Siopa, Michele Melchiorre $\mathbb{B}^{\circ}$, and Susanne Siebentritt \\ Laboratory for Photovoltaics, Physics and Materials Science Research Unit, University of Luxembourg, \\ 44 Rue du Brill, 4422 Belvaux, Luxembourg
}

(Received 10 July 2020; revised 16 March 2021; accepted 23 April 2021; published 21 May 2021)

\begin{abstract}
$\mathrm{Cu}(\mathrm{In}, \mathrm{Ga}) \mathrm{S}_{2}$ is a promising semiconductor that offers excellent prospects for photovoltaics. However, the performance has been plagued mostly due to large photovoltage deficit. Here, we investigate defects and optoelectronic properties of $1.7-\mathrm{eV}$ band gap near-stoichiometric $\mathrm{Cu}(\mathrm{In}, \mathrm{Ga}) \mathrm{S}_{2}$ (CIGS). We have estimated quasiFermi-level splitting of $921 \mathrm{meV}$ from steady state photoluminescence (PL) measurements at 1 sun. Detailed analysis of temperature and excitation dependent PL reveals the behavior of a strongly compensated semiconductor. We show that spatially varying energetic disorder, described by electrostatic potential fluctuations, causes band-tail recombination and strongly affects the carrier recombination in compensated CIGS. Apart from band-tail transitions, we also observe two deep defects at about 0.3 and $0.45 \mathrm{eV}$ below the band edge. The defects are also discerned by admittance measurements. Temperature dependent current-voltage measurements show that the open-circuit voltage is further limited by interface recombination. Thus, the performance improvement lies in the mitigation of deep defect states and absorber/buffer interface optimization.
\end{abstract}

DOI: 10.1103/PhysRevMaterials.5.055403

\section{INTRODUCTION}

The field of photovoltaics (PV) has witnessed considerable advancements in the past few decades leading to material innovations, advanced engineering, and improved fundamental understanding. Crystalline silicon, thin film $\mathrm{Cu}(\mathrm{In}, \mathrm{Ga})(\mathrm{S}, \mathrm{Se})_{2}$ (CIGSSe) and perovskite single junction solar cell record efficiencies have reached $27.6 \%, 23.4 \%$, and $25.2 \%$ respectively [1-3]. Among thin film technologies, chalcopyrites based on CIGSSe have shown remarkable prospects for high efficiency thin film and flexible solar cells along with good stability [4,5]. In addition to that, composition variation allows band gap tuning making chalcopyrites extremely useful for tandem solar cells. The performance improvements in these individual technologies have drawn considerable attention on tandem solar cells to further boost efficiencies. The high power conversion efficiencies from tandem solar cells are desirable from a cost perspective and long term deployment as it reduces the energy payback time [6].

Tandem solar cells offer higher efficiencies than single junction solar cells by better utilizing the solar energy spectrum. A combination of 1.1-1.2 eV lower band gap bottom cell with 1.7-1.8 eV wide band gap top cell has a potential

\footnotetext{
*Corresponding author: sudhanshu.shukla@uni.lu; sudhanshu. shukla2409@gmail.com

Published by the American Physical Society under the terms of the Creative Commons Attribution 4.0 International license. Further distribution of this work must maintain attribution to the author(s) and the published article's title, journal citation, and DOI.
}

to achieve $>30 \%$ power conversion efficiency [7]. Highly efficient low band gap semiconductors such as $\mathrm{Si}, \mathrm{CuInSe}_{2}$

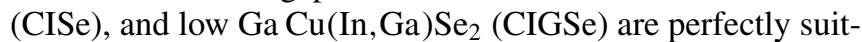
able for bottom cells. On the other hand, top cell candidates include halide perovskites, CIGSe (with high Ga content), and pure sulfide CIGS. Even though selenide based CIGSSe has achieved efficiencies beyond $23 \%$ [4], the tandem prospects for CIGSe are not quite favorable due to limited band gap tunability by Ga alloying (1.0-1.6 eV). Wide band gap CGSe has been used in tandem configurations with low band gap CIGSe solar cells [8]. CuInGaS 2 based chalcopyrite is an excellent absorber for developing a top cell for tandem applications owing to its broad band gap tunability from 1.5 $\left(\mathrm{CuInS}_{2}\right)$ to $2.4 \mathrm{eV}\left(\mathrm{CuGaS}_{2}\right)$ [9-11]. However, the power conversion efficiency remained considerably lower than its theoretical maximum due to the problem of low photovoltage [12-14]. For $\mathrm{CuInS}_{2}$, Lomuscio et al. demonstrated that the quasi-Fermi-level splitting (QFLS) deficit with respect to band gap, a quantity directly correlated with the quality of the bulk absorber, is significant and predominantly limited by deep defects [15]. Moreover, photovoltage deficit is found to increase with respect to the band gap as the amount of $\mathrm{Ga}$ is increased [13]. Therefore, it remained challenging to realize an efficient high band gap CIGS. Proposed explanations include the existence of phase impurities $[16,17]$, conducting $\mathrm{Cu}-\mathrm{S}$ related phases [18], recombination losses due to high concentration of intrinsic bulk point defects (particularly extended defect states due to sulfur vacancies and cation antisite defects) $[15,19]$, back surface recombination at Mo and absorber/buffer interface (front surface) [20-23]. This indicates that the key to higher QFLS, and hence $V_{\mathrm{oc}}$, lies in mitigating the defects and improving the optoelectronic quality of 
the absorber. In addition to that, recombination at the buffer interface severely affects the photovoltage and requires a judicious choice of buffer layer to minimize deleterious band offsets to address interface recombination losses. To date, most of the efficient devices are made using a CIGS absorber grown under $\mathrm{Cu}$-excess conditions (Cu-rich) [14,24]. However, the most recent reports on sulfide CIGS processed under $\mathrm{Cu}$-deficient conditions spurred a renewed interest with efficiencies reaching $>15 \%$, and photovoltage approaching $1 \mathrm{~V}$ $[12,25]$. Nevertheless, the photovoltage remained lower with high $V_{\mathrm{oc}}$ deficit. In chalcopyrite, optical transitions critically depend on the composition. Notable studies have focused on the optical transitions and the nature of recombination for pure CIS and CGS [19,26-29], but much remains to be understood for alloyed CIGS. Therefore, it is imperative to understand the intrinsic defects and charge carrier recombination process in CIGS to reduce the recombination losses.

In this paper, we investigate recombination processes, through comprehensive temperature and excitation dependent $\mathrm{PL}$ and admittance spectroscopy, in high quality slightly $\mathrm{Cu}-$ deficient or near-stoichiometric CIGS prepared by a single stage co-evaporation process. The absorber was characterized by X-ray diffraction (XRD), scanning electron microscopy (SEM), and Raman measurements for crystallinity and phase purity. Photoluminescence (PL) spectroscopy is an extremely sensitive and nondestructive technique to probe the defects [30-32]. QFLS can be determined from absolute calibrated PL analysis, which serves as a quality metric of the absorber and represents the upper limit of the photovoltage [33-36]. We determined a QFLS value of $921 \mathrm{meV}$ at 1-sun equivalent illumination from calibrated photoluminescence measurements. We were able to identify defect transitions characteristic to band tails and deep-defect assisted recombination. We suggest the origin of tail states due to electrostatic potential fluctuations arising from random spatial variations in defect density. These fluctuations influence the PL transition which are identified in terms of energy shift of the PL maximum with excitation and temperature. We validated the energetic position of the defect states from temperature dependent capacitance measurements. The bulk recombination processes severely limit the QFLS of the performance of our stoichiometric CIGS absorber. Devices with a CdS buffer layer demonstrated a photovoltage of $746 \mathrm{mV}$, less than QFLS of the bare absorber, indicative of additional interface recombination losses.

\section{EXPERIMENTAL DETAILS}

$\mathrm{CuInGaS}_{2}$ (CIGS) absorbers were deposited on Mo coated soda-lime glass (SLG) by single stage co-evaporation process in a physical vapor deposition (PVD) system. Cu, In, Ga, and $\mathrm{S}$ were simultaneously co-evaporated with constant flux of individual sources maintained throughout the deposition process. The substrate was held at constant temperature of $\sim 510^{\circ} \mathrm{C}$, and at constant rotation of 4 RPM for the entire 1-h deposition duration. Subsequently, substrates were allowed to cool down naturally in the presence of sulfur vapor in order to maintain sulfur overpressure and thus avoid any sulfur loss from the film. The composition of the absorber was determined from energy dispersive x-ray spectroscopy (EDS) after etching in $5 \% \mathrm{KCN}$ solution for $30 \mathrm{sec}$. The
$[\mathrm{Cu}] /[\mathrm{In}+\mathrm{Ga}]$ ratio of the absorber was close to 0.98 . $\mathrm{X}$-ray diffraction measurements were performed using a diffractometer with $\mathrm{Cu}-K_{\alpha}$ radiation in $\theta-2 \theta$ Bragg-Brentano geometry. Raman measurements were performed with a micro-Raman spectrometer using a laser excitation of 532 $\mathrm{nm}$ at a power of $0.2 \mathrm{~mW}$ and spot size of $0.9 \mu \mathrm{m}$. QFLS was derived from absolute intensity photoluminescence spectrum using the generalized Planck's law [35]. Briefly, the QFLS was obtained by fitting of high energy slope of the photoluminescence spectra at energy sufficiently larger than the band gap, assuming absorptivity value of 1 . Low temperature photoluminescence measurements were carried out in a home-built system equipped with a helium flow cryostat under 663-nm wavelength diode laser excitation source. The emitted luminescence was collected through parabolic mirrors focused into a fiber, and spectrally resolved by a monochromator and detected by a Si-charge-coupled device (CCD) and an InGaAs diode array. All the data are corrected with a calibration lamp with a known spectrum. Neutral density filters were used to vary the power for excitation intensity dependent PL. The setup allows a wavelength resolution in the visible of about $2 \mathrm{~nm}$. Carrier lifetime measurements performed using a time-correlated single photon counting (TCSPC) system. Laser excitation of wavelength $640 \mathrm{~nm}$ with a repetition rate of $20 \mathrm{MHz}$ and detection bandwidth of $45 \mathrm{~nm}$ around $750 \mathrm{~nm}$ was used.

Solar cells were fabricated from the absorber by successive layers of chemical bath deposited $\mathrm{CdS}$ buffer layer, sputtered intrinsic $\mathrm{ZnO}$ and $\mathrm{Al}: \mathrm{ZnO}$ window layer and $e$-beam evaporated $\mathrm{Ni} / \mathrm{Al}$ metal contact grids. Solar cell characterization was performed using a class AAA solar simulator under simulated AM 1.5 radiation and external quantum efficiency (EQE) using chopped illumination from a halogen/xenon lamp and a lock-in amplifier to measure the photocurrent. Temperature dependent $J V(J V T)$ measurements were performed in the temperature range 320-50 K using a closed-cycle helium cryostat. The short-circuit current density $\left(J_{\mathrm{sc}}\right)$ measured from the $J V$ set-up under the standard condition was used to set the illumination intensity for the JVT measurements. Dark and light measurements were recorded using a mechanical shutter. Admittance measurements were performed using a precision LCR meter in the same JVT setup. As discussed in Sec. III, additional characterization results are provided in the Supplemental Material [37].

\section{RESULTS AND DISCUSSION}

\section{A. Structural characterization and phase analysis}

The chemical composition of the film was analyzed by EDX which showed a $\mathrm{Cu}: \mathrm{In}+\mathrm{Ga}$ ratio close to 0.98 and a $\mathrm{Ga}: \mathrm{Ga}+\mathrm{In}$ ratio to be around 0.30 . Structural characterization was performed by XRD analysis of polycrystalline CIGS thin films. Figure 1(a) shows the $\theta-2 \theta$ diffractogram plots for CIS, CGS, and CIGS thin films.

In order to compare the XRD peak shift, we measured pure CIS and CGS films grown by a similar process to serve as a reference, as shown in Fig. 1(a). All diffraction peaks could be indexed to chalcopyrite-type structure with reference to the ICSD database no. 186714 and no. 28736 for 

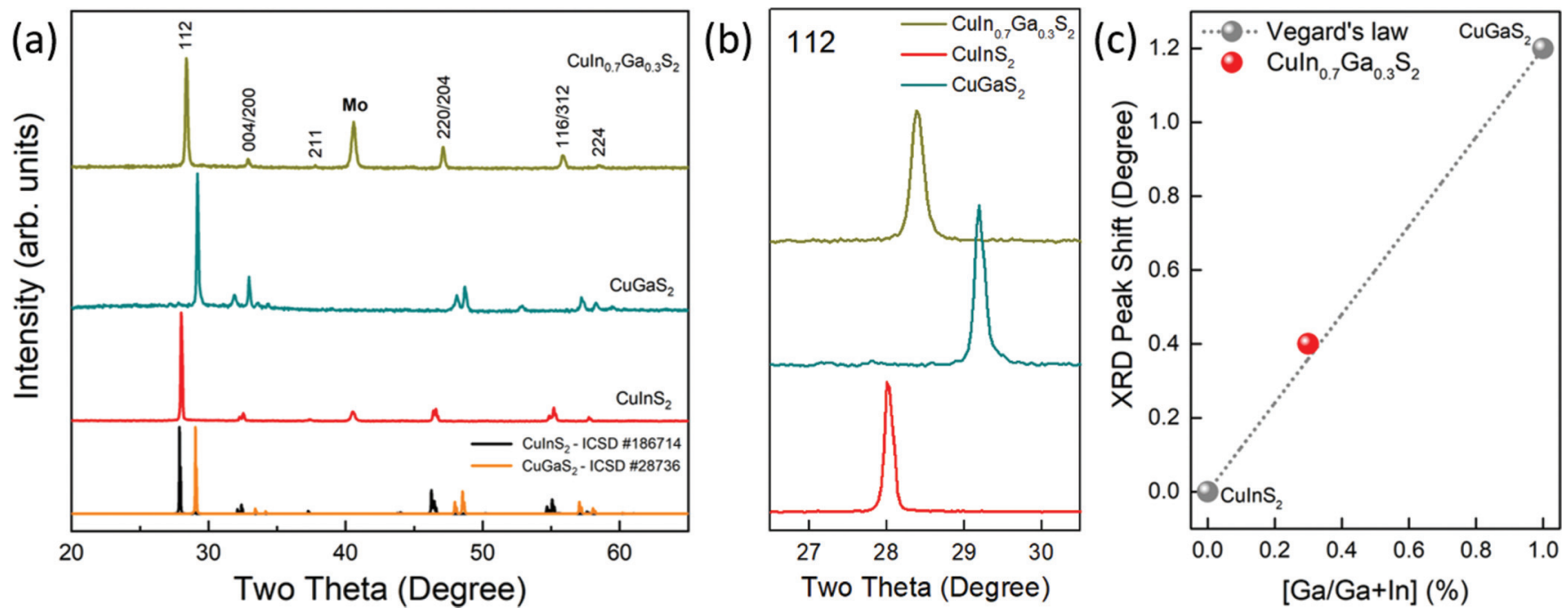

FIG. 1. (a) XRD of CIGS, CIS, and CGS thin film on Mo substrate. (b) 112 peak position shift with Ga content in the film and (c) XRD peak shift obtained from (b) as a function of $\mathrm{Ga} / \mathrm{Ga}+\mathrm{In}$ ratio; dotted line represents the linear function predicted by Vegard's law.

pure $\mathrm{CuInS}_{2}$ and $\mathrm{CuGaS}_{2}$ respectively. CIGS crystallizes in tetragonal chalcopyrite structure similar to CIS (space group $I \overline{4} 2 d)$. All three films showed the highest diffraction peak intensity for the (112) plane. Upon addition of Ga, diffraction peaks shift to higher angles due to lattice contraction resulting from the smaller $\mathrm{Ga}$ atoms partially occupying the In lattice sites, as shown in Fig. 1(b). According to Vegard's law [38], a first order linear relationship is expected between the shift in the diffraction peak and stoichiometry variation, as $x$ in $\mathrm{CuIn}_{x} \mathrm{Ga}_{1-x} \mathrm{~S}_{2}$ varies from 1 (CIS) to 0 (CGS). The XRD peak shift for the $[\mathrm{Ga}] /[\mathrm{Ga}+\mathrm{In}](\mathrm{GGI})$ ratio of 0.3 lies in a straight line consistent with Vegard's law [Fig. 1(c)].

Figure 2(a) shows the SEM topview and cross-section micrographs of the $750 \pm 50 \mathrm{~nm}$ thick CIGS film. A rough surface morphology appears with well-connected columnar grains, similar to the films reported in the literature [15], for single stage deposited CIGS thin films. While columnar grains are favorable for charge transport, grain boundaries lying normal to the substrate might provide shunting pathways and have serious repercussions on the device performance. Conducting $\mathrm{Cu}-\mathrm{S}$ phases and complex CIGS phase impurities, especially in the Cu-poor domain, are detrimental and need to be examined closely [39]. These secondary phases, especially $\mathrm{CuIn}_{5} \mathrm{~S}_{8}$, might exist in too small a quantity to efficiently diffract $x$ rays and eventually evade in the XRD measurements, making their identification difficult. Raman spectroscopy has proven to be a useful tool for probing phase impurities. Figure 2(b) shows the Raman spectrum of the CIGS film on Mo substrate. The prominent Raman band at $296 \mathrm{~cm}^{-1}$ corresponds to the characteristic $A 1$ mode of chalcopyrite phase $(\mathrm{CH})$ consistent with the reported value in the literature [40]. For pure CIS and CGS, the A1 mode normally appears at 291 and $311 \mathrm{~cm}^{-1}$ respectively. The chalcopyrite $A 1$ vibrational mode shifts to higher wave numbers (a)
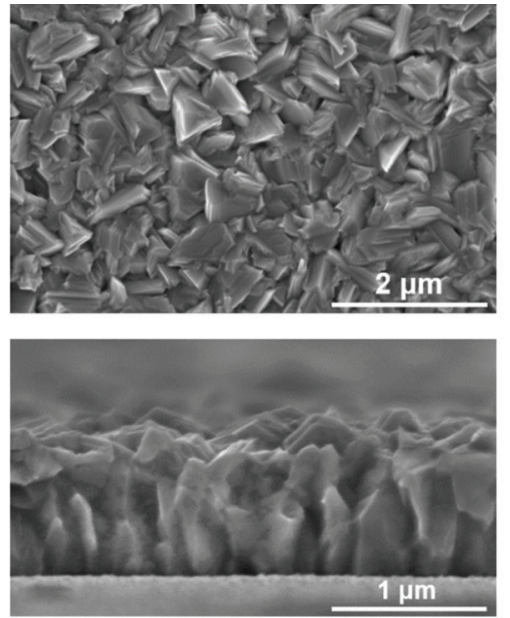

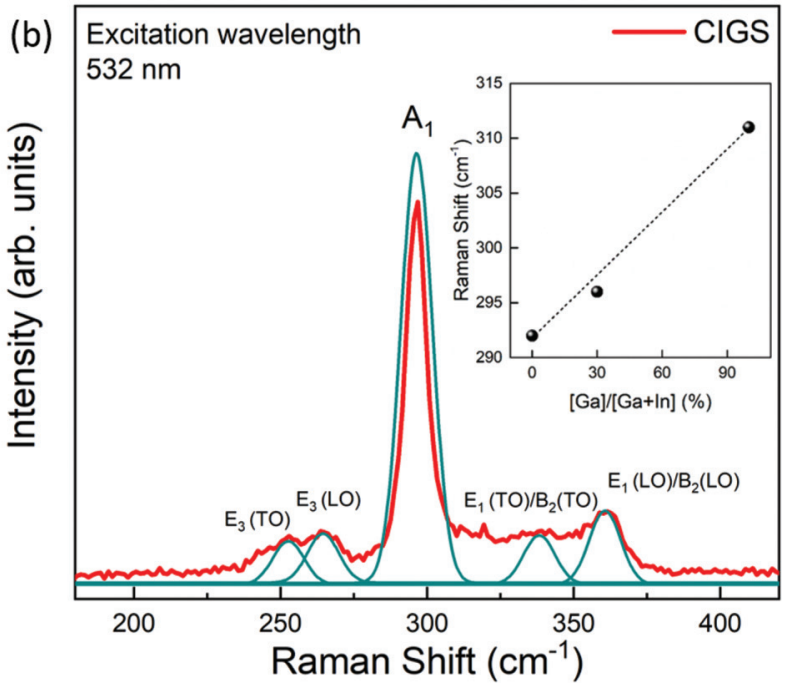

FIG. 2. (a) Top-view and cross-section SEM micrograph of CIGS thin film deposited on Mo substrate. (b) Raman spectrum of the CIGS film on Mo substrate. 

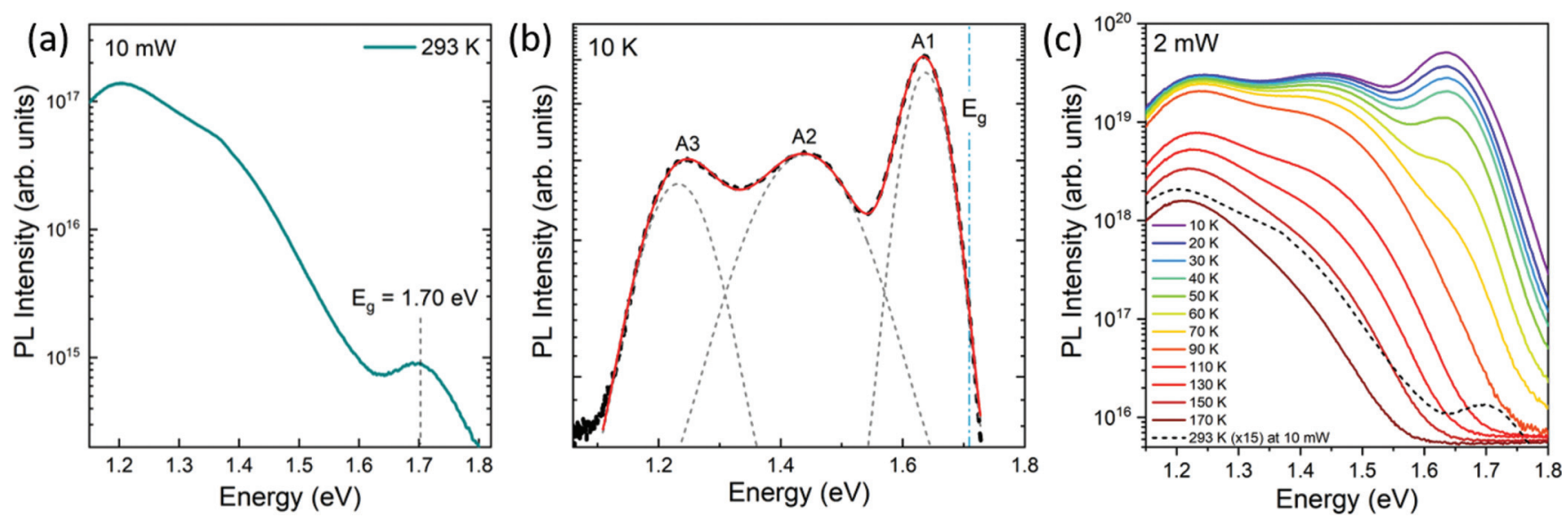

FIG. 3. Photoluminescence spectra of the CIGS thin film on Mo substrate (a) at room temperature and (b) 10 K. (dashed line corresponds to the band gap of the absorber at room temperature) and (c) temperature dependent photoluminescence spectra of the CIGS thin film.

as the amount of $\mathrm{Ga}$ increases. The $A 1$ mode shifts to $296 \mathrm{~cm}^{-1}$ for GGI of 0.3 , as shown in the inset of Fig. 2(b). Fitting the Raman spectra yields additional modes at 252, 264,338 , and $360 \mathrm{~cm}^{-1}$ corresponding to $E_{3}(\mathrm{TO}), E_{3}(\mathrm{LO})$, $E_{1}(\mathrm{TO}) / B_{2}(\mathrm{TO}), E_{1}(\mathrm{LO}) / B_{2}(\mathrm{LO})$ vibrational modes respectively $[41,42]$. No distinct peak related to the $\mathrm{Cu}-\mathrm{Au}$ (CA) phase $\left(307 \mathrm{~cm}^{-1}\right)$ can be identified. However, it is possible that the peak contribution is too small and may be eventually obscured by the $A 1$ peak. Thus, combined analysis of XRD and Raman measurements demonstrate the phase pure chalcopyrite film.

\section{B. Photoluminescence and defect analysis}

Investigations on the electronic structure of the CIGS film was performed by PL spectroscopy. We conducted room temperature and detailed excitation and temperature dependent photoluminescence measurements to study the electronic defects in CIGS. As shown in Fig. 3(a), the room temperature PL spectrum shows a peak at $1.70 \mathrm{eV}$ corresponding to the band gap $\left(E_{g}\right.$ of $1.72 \mathrm{eV}$ obtained from the EQE, described later in the discussion) and an additional broad emission (BE) appears at lower energy due to in-gap defect states. The higher intensity of the BE indicates predominant recombination through defect states in the gap. Quasi-Fermi-level splitting (QFLS) of $921 \mathrm{eV}$ is obtained from the main PL peak. The presence of deep defects causes excess carrier recombination losses within the bulk and limit the QFLS, resulting in a high QFLS deficit with respect to the band gap. Analysis of low temperature PL provides detailed information about the discrete PL transitions and the basic nature of specific recombination processes. Figure 3(b) shows the PL spectrum of the absorber at $10 \mathrm{~K}$ with laser incident power of $2 \mathrm{~mW}$, indicated by three optical transitions labeled as $A 1$ (close to the band edge) and defect related lower energy peaks as $A 2$ and $A 3$. Figure 3(c) shows the PL peak evolution with increasing temperature. The two notable observations are, first, the rapid quenching and eventual disappearing of the main PL peak (A1) leaving PL emission appearing mainly from the deep localized defect states $A 2$ and $A 3$ (see also Fig. S1 of the Supplemental Material [37]); second, the overall PL intensity reduces due to higher nonradiative recombination, as expected for luminescence in semiconductors [43].

The main PL emission at $\sim 1.6 \mathrm{eV}(A 1)$ appears at energy lower than the room temperature band gap value $(1.7 \mathrm{eV})$. This significant redshifted luminescence implies recombination through band-tail or defect related transitions. A first hint on the nature of PL transitions can be obtained by examining the exponent $k$ in the power law relationship,

$$
I \propto \varphi^{k},
$$

where $I$ and $\varphi$ are the PL intensity and laser excitation power respectively. In the absence of deep defects, the value of $k>1$ infers band-to-band or excitonic recombination and $k<1$ is attributed to defect related recombination [44]. However, if the deep defects are present, the assignment based on the $k$ value becomes nontrivial due to the competing recombination processes [45]. In addition to PL intensity, PL peak energy shift (represented by $\beta$ as peak-shift/decade) also allows us to identify different recombination mechanisms based on their characteristic dependence on incident laser power. The dependence of the PL peak position as a function of excitation can be expressed by the following empirical equation [43]:

$$
E_{\mathrm{PL}}\left(I_{\mathrm{ex}}\right)=E_{\mathrm{PL}}\left(I_{o}\right)+\beta \log _{10}\left(\frac{I_{\mathrm{ex}}}{I_{o}}\right),
$$

where $E_{\mathrm{PL}}\left(I_{\mathrm{ex}}\right)$ is the PL peak energy position at higher excitation intensity and $E_{\mathrm{PL}}\left(I_{\mathrm{O}}\right)$ is the unperturbed PL peak position.

Notably, we found that the low energy peaks ( $A 2$ and $A 3)$ are affected by interferences, while the peak $A 1$ remains unaffected. This was confirmed by measuring the PL after modifying the absorber surface with a thin polystyrene scattering layer [46,47], which enables us to obtain interference-free PL (see Fig. S2 [37]). Since the energy position and the height of the defect related peaks is influenced by interference shifts, we cannot analyze them in more detail. Therefore, we focus only on the main peak $A 1$ for the rest of the PL analysis.

Figures 4(a) and 4(b) show the power dependent PL measurements at $10 \mathrm{~K}$. At $5 \mathrm{~K} \mathrm{PL}$ peak broadening is observed (or it could be a shoulder peak as shown by the dotted line in Fig. S3 [37]). Therefore, we focused on the $10 \mathrm{~K}$ spectra. We found a $k$ value of 1.4 for the $A 1$ peak from Eq. (1) (intensity $I$ was obtained using peak heights of the Gaussian fits of 

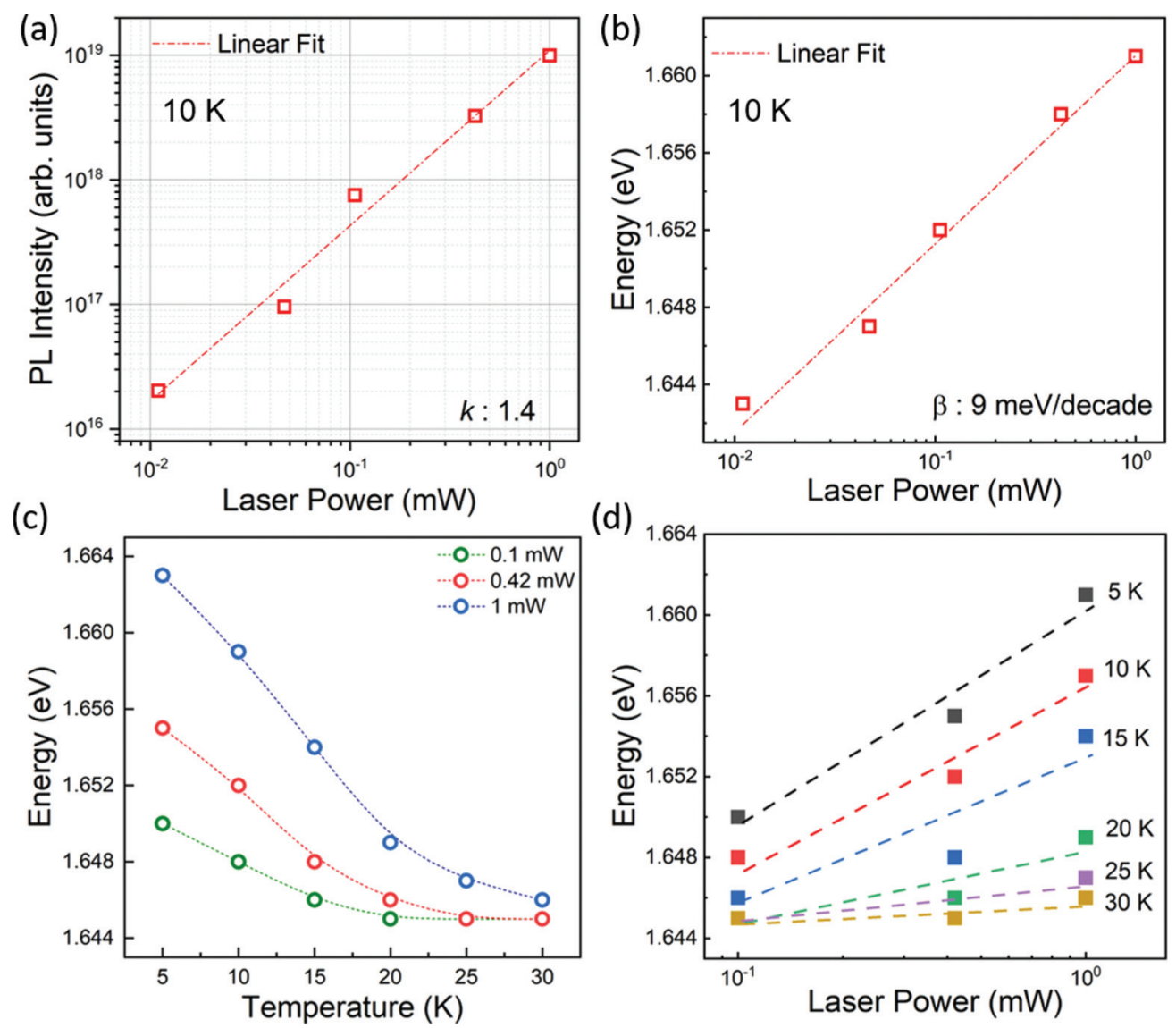

FIG. 4. Laser excitation dependent (a) PL peak intensity and (b) PL peak position. (c) Temperature dependence of PL peak position at different laser excitation power. The lines are a guide to the eye (peak positions are obtained from the Gaussian fitting of the spectra). (d) Laser excitation dependent PL peak position at different temperatures.

the spectra). $A 2$ and $A 3$ peaks show $k<1$, which, together with lower energy of PL emission, indicates defect related transition (see Supplemental Material Fig. S4 [37]). The value of $k>1$ for $A 1$ indicates the involvement of band states. On increasing the power, the $A 1$ PL peak exhibits a blueshift with a $\beta$ value of $9 \mathrm{meV} /$ decade, from Fig. 4(b). In the context of the existing models of recombination mechanism [30,4850], the $\beta$ value of $9 \mathrm{meV} /$ decade is not consistent with the standard donor-acceptor pair (DAP) transition, for which a blueshift of a few meV/decade is expected. The higher blueshift with excitation along with PL peak position lower than the band gap observed in our case could be understood in terms of band-tail related recombination. In the case of high compensation, electrostatic potential fluctuations due to unscreened spatially varying densities of charged donor and acceptor defects are known to dominate the recombination process in chalcopyrites [48]. Electrostatic potential fluctuations cause band tails and have been observed in kesterites and $\mathrm{Cu}$-poor CIGSe and CGSe [51-54]. As the excitation power is increased, photogenerated free carriers result in the reduction of potential fluctuations due to the defect neutralization and enhanced screening of the charged defects. Thus, photoexcited carriers populate higher energy states where density of states is available in the band-tail region. It is to be noted that band-tail and tail-tail recombination mechanisms are intermixed and difficult to distinguish.
More evidence in favor of band-tail recombination comes from temperature dependent PL measurements. We investigated temperature dependent PL at different excitation fluence to shed light on the distribution of tail states. PL spectra display rapid quenching of $A 1$ as the temperature is increased (see also Fig. S1 [37]). Figure 4(c) shows the temperature dependent PL of the $A 1$ peak at $0.1,0.42$, and $1 \mathrm{~mW}$ laser power. The PL peak position of transition $A 1$ shows a redshift with increasing temperature. The observed trend is expected for band-tail recombination [55-57], consistent with the models proposed for $\mathrm{Cu}$-poor chalcopyrites and other heavily doped and strongly compensated semiconductors $[48,53,58,59]$. At the lowest temperature, carriers are randomly distributed across the density of tail states. As the temperature is increased, sufficient energy becomes available to the carriers to hop across the fluctuating potential barriers and build up carrier population in the deeper lying unoccupied states, resulting in a redshift of the PL maximum. The blueshift with laser excitation continuously reduces as the temperature is increased, as shown in Fig. 4(d), indicates the flattening of the potential fluctuations. The PL transients, measured at room temperature, reveal short carrier recombination lifetime of $\sim 220$ ps for band-band transition (see also Fig. S5 [37]). Such short recombination lifetime reflects significant nonradiative recombination in the material. In contrast, the lower energy defect peak at $1.2 \mathrm{eV}$ yields a longer luminescence 

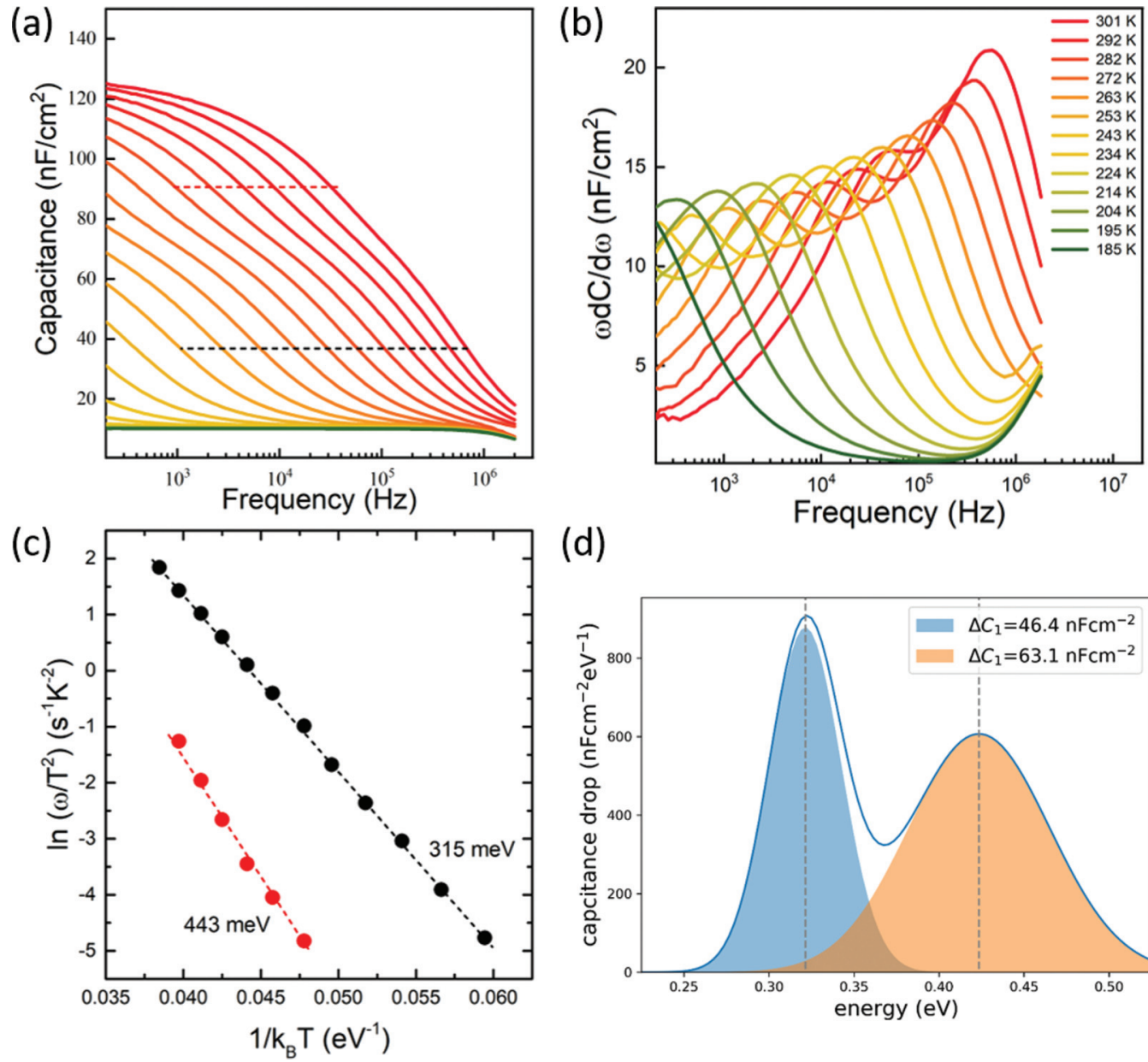

(d)

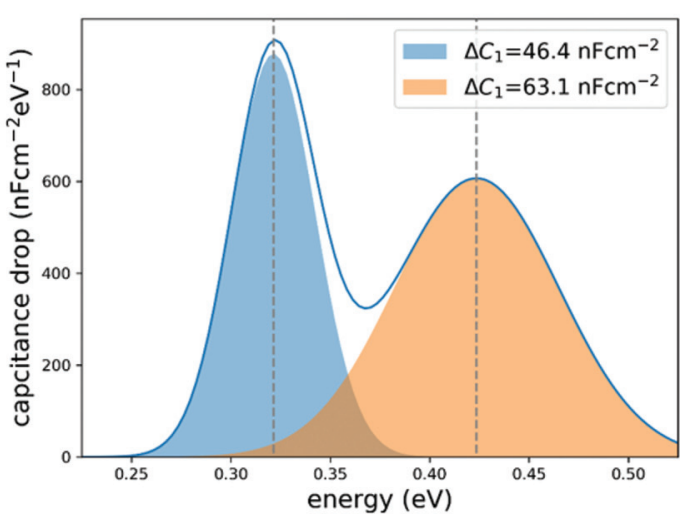

FIG. 5. (a) Frequency dependent capacitance measurement of the CIGS solar cell at various temperature from 300 to $180 \mathrm{~K}$. Capacitance steps are depicted by dashed lines. (b) Logarithmic capacitance derivatives of the same measurements, clearly indicating two inflection points. (c) Arrhenius plots for corresponding capacitance steps and (d) analysis of defect distribution shown as capacitance drop according to [62].

lifetime of $\sim 3$ ns indicative of localized deep trap mediated recombination. Based on these results, we see that the carrier recombination in stoichiometric CIGS is dominated by band-tail recombination and deep defects. A model for carrier recombination is presented later.

In order to understand the nature of the deep defects $A 2$ and $A 3$, we carried out capacitance measurements on the full device. Capacitance measurements were performed on the full device with a $\mathrm{CdS}$ buffer layer and a $\mathrm{ZnO}$ window. Steps in capacitance spectra are usually interpreted as the contribution of defect level within the space charge region (SCR). However, interface defects and transport barriers might also contribute to the capacitance signal and therefore they must be interpreted with caution $[60,61]$.

Figure 5(a) shows the frequency dependent capacitance spectra of the device measured at different temperatures. Two capacitance steps are observable in the spectra and corresponding inflection points are shown by the dotted lines. These inflection frequencies were obtained from the maxima of the capacitance derivative with respect to $\log$ (frequency) in Fig. 5(b). Arrhenius plots corresponding to the inflection frequencies are shown in Fig. 5(c). Activation energy of 315 and $443 \mathrm{meV}$ is obtained from the slope of the Arrhenius plots. In order to ascertain whether these capacitance features are indeed due to defect responses and not from a transport barrier, we analyzed temperature dependent series resistance and dark diode current from IVT measurements. Lower activation energy of the series resistance from IVT measurements support the origin of the capacitance steps due to defects. When the defect energies are not discreet but show a broad density of states, a density of states analysis of the admittance spectra is more appropriate. Approximating Gaussian-shaped energy distribution of deep defect states and using the method described in Ref. [62], we obtained defect distribution and corresponding maxima of the defect DOS at 321 and $424 \mathrm{meV}$, as shown in Fig. 5(d). The energy level positions of the defects are consistent with the defect energies obtained from Arrhenius plots. The $424-\mathrm{meV}$ defect is characterized a by broader energy distribution with standard deviation of $42 \mathrm{meV}$ compared to $21 \mathrm{meV}$ for the 321-meV defect. A larger change in the capacitance drop $(\Delta C)$ for the $424-\mathrm{meV}$ defect indicates a higher defect density of this particular defect. The energetic position of the defects within the band gap can be approximated from a known room temperature band gap from PL and defect energy obtained from capacitance. Thus, the defect levels corresponding to 321 and $424 \mathrm{meV}$ makes a recombination likely with energies $\sim 1.38$ and $1.26 \mathrm{eV}$ respectively. These values are roughly compatible with the $A 2$ and $A 3 \mathrm{PL}$ 

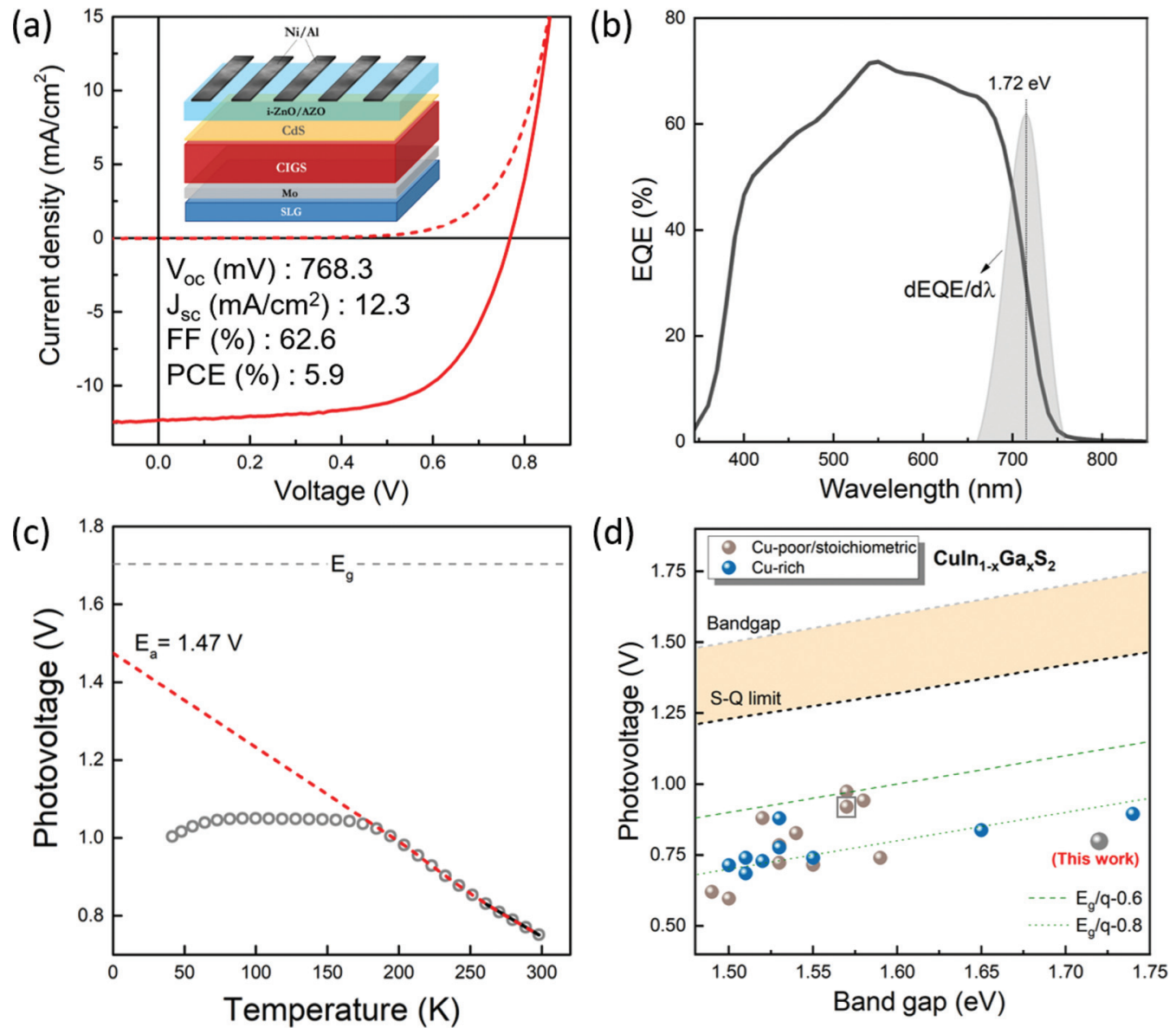

FIG. 6. (a) Current-voltage ( $J V$ ) characteristics of CIGS solar cell measured in the dark and under 1-sun illumination. (b) External quantum efficiency (EQE) of the corresponding device. (c) Temperature dependent open-circuit voltage and extracted activation energy at $0 \mathrm{~K}$ obtained from linear fit. (d) Photovoltage obtained from various CIGS (Cu-rich and $\mathrm{Cu}$-poor) solar cells reported in the literature [12-14,20,25,63-73] with different band gap values including the current work. Record solar cell data are shown in the box.

peaks. Thus, PL and capacitance measurements corroborate the presence of two deep defects at about 300 and $400 \mathrm{meV}$, respectively, from the band edge.

\section{Device performance and analysis}

We measured the performance of the CIGS solar cell with $\mathrm{CdS}$ buffer layer. $J V$ characteristics in the dark and under illumination are shown in Fig. 6(a) along with electrical parameters; corresponding diode fit values are listed in Fig. S6 [37]. $V_{\text {oc }}$ deficit with respect to the band gap $\left(E_{\mathrm{g}}-V_{\mathrm{oc}}\right)$ is calculated to be around $930 \mathrm{meV}$. Notably, Larsson et al. recently reported a $V_{\text {oc }}$ deficit of around $906 \mathrm{meV}$ for 1.63-eV band gap $\mathrm{CuGaSe}_{2}$ solar cells with CdS buffer layer [74]. Figure 6(b) shows the EQE of the solar cell reaching a maximum value of $71 \%$ at $550 \mathrm{~nm}$. The band gap value was obtained from the inflection point of the low energy onset of the EQE spectrum. The band gap value from EQE $(1.72 \mathrm{eV})$ is consistent with the PL band gap (1.70 eV). The lower EQE values at shorter wavelengths indicate losses near the CIGS/CdS interface. $V_{\mathrm{oc}}$ is also limited by absorber/buffer interface loss which is manifested by the $V_{\text {oc }}$ deficit of $153 \mathrm{meV}$ with respect to the QFLS value. Interface recombination could be reduced by an alternative buffer layer better matched for band offsets.
The integrated current density of $13.2 \mathrm{~mA} / \mathrm{cm}^{2}$ from EQE measurements is slightly higher than the $J_{\text {sc }}$ from $J V$ measurements, due to spectral mismatch of the solar simulator. Temperature dependent $J V(J V T)$ measurements are shown in Fig. 6(c) to identify the dominant recombination mechanism in the solar cell. The extrapolated activation energy at $0 \mathrm{~K}$ is substantially lower $(1.47 \mathrm{eV})$ than the band gap, implying interface recombination to be the limiting factor, which is expected for the CdS buffer layer devices due to a negative conduction band offset between absorber and buffer [25]. This stresses the requirement of a buffer layer with suitable energy band alignment with CIGS to reduce the photovoltage loss and consequently enhance the overall performance. QFLS is a useful metric to quantify the quality of the absorber and is the maximum limit of the photovoltage that could be achieved from the absorber. Measurement on the bare absorber on Mo substrate yields a QFLS value of $921 \mathrm{meV}$, significantly lower than the band gap, which signifies recombination losses in the bulk due to defects. Mitigation of bulk defects would enhance the QFLS value. Figure 6(d) shows the photovoltage values reported in the literature and the current work, including the record cell marked in square (15.5\%, Solar Frontier) [25]. It is evident that CIGS solar cells suffer from higher photovoltage deficit compared to Se-based CIGSe solar cells. The 


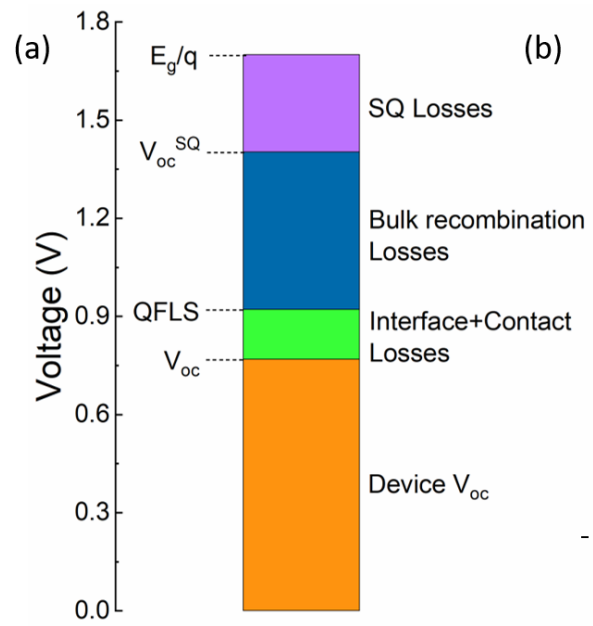

(b)

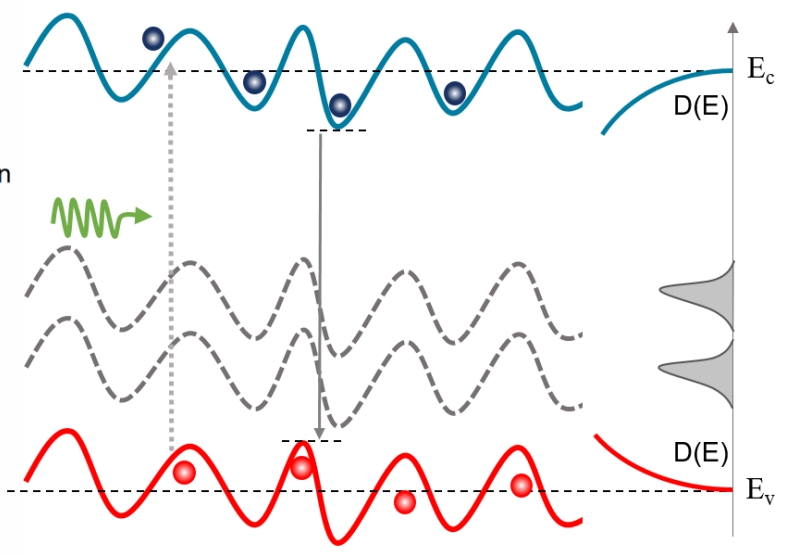

FIG. 7. (a) $V_{\text {oc }}$ loss analysis for CIGS solar cell with CdS buffer layer. (b) Recombination model for CIGS film based on PL and capacitance measurements; energy levels shown in dashed lines represent deep defects.

higher photovoltage deficit values reported for pure CIS solar cells are due to the fact that majority of the chalcopyrite films being $\mathrm{Cu}$-rich or close to stoichiometry, which suffer from high surface recombination. A Cu-poor surface is desirable to suppress the interface recombination loss. However, the thermodynamic instabilities inhibit the formation of pure chalcopyrite phase in the $\mathrm{Cu}$-poor domain [39]. High photovoltage from $\mathrm{Cu}$-poor $\mathrm{CIGS}$ films suggest that $\mathrm{Ga}$ addition might be helpful in stabilizing the chalcopyrite phase and allow us to explore wider composition range in the $\mathrm{Cu}$-poor domain $[25,73,75]$. Nevertheless, the photovoltage loss remains higher for wide band gap CIGS. This could be due to the higher likelihood of deep defects in wide band gap chalcopyrite [32]. Evidently, there is still a lot of room left for improvement in the bulk and interface loss minimization. Figure 7 summarizes the photovoltage losses in the CIGS solar cell studied in this work. The solar cell performance is severely limited by the bulk recombination losses and additional losses occur at the interface. To reduce the gap between $V_{\text {oc }}{ }^{\mathrm{SQ}}$ and QFLS, deep defects need to be suppressed. Further buffer layer optimization with reduced interface losses would help in enabling $V_{\text {oc }}$ close to QFLS.

\section{CONCLUSION}

In summary, we present a detailed analysis of recombination processes in $1.7-\mathrm{eV}$ band gap CIGS. We found that the recombination in stoichiometric absorber is dominated by band tails induced by fluctuating potentials, manifested by the temperature and excitation dependent analysis of the PL at low temperature. The transitions are strongly influenced by potential fluctuations, resulting in a higher blueshift with excitation and redshift with temperature increase. Additionally, we observe two dominant deep defect PL peaks, which are also confirmed by admittance measurements. The presence of deep defects introduces significant nonradiative losses within the bulk, which accounts for major $V_{\text {oc }}$ losses. Finally, we demonstrate solar cells with photovoltage of $768 \mathrm{mV}$, less than the QFLS value of $921 \mathrm{meV}$, indicating interface recombination in addition to bulk related losses. This indicates that the improvement in photovoltage is possible through avoiding the formation of deep defect and potential fluctuations in the bulk and also manipulating the interface to reduce losses at the buffer/absorber interface.

\section{ACKNOWLEDGMENTS}

The authors gratefully acknowledge the Luxembourg Fonds National de la Researche (FNR) for funding under SeVac (C17/MS/11655733) and CorrKest (C15/MS/10386094) projects. M.S., D.S., and D.A. thank the support and funding from MASSENA doctoral program (FNR PRIDE/15/10935404). The authors thank the reviewer (anonymous) for his insightful comments on the work.
[1] https://www.nrel.gov/pv/cell-efficiency.html.

[2] M. A. Green and A. W. Y. Ho-Baillie, ACS Energy Lett. 4, 1639 (2019).

[3] M. A. Green, E. D. Dunlop, J. Hohl-Ebinger, M. Yoshita, N. Kopidakis, and A. W. Y. Ho-Baillie, Prog. Photovoltaics Res. Appl. 28, 3 (2020).

[4] M. Nakamura, K. Yamaguchi, Y. Kimoto, Y. Yasaki, T. Kato, and H. Sugimoto, IEEE J. Photovoltaics 9, 1863 (2019).

[5] R. Carron, S. Nishiwaki, T. Feurer, R. Hertwig, E. Avancini, J. Löckinger, S.-C. Yang, S. Buecheler, and A. N. Tiwari, Adv. Energy Mater. 9, 1900408 (2019).
[6] T. Leijtens, K. A. Bush, R. Prasanna, and M. D. McGehee, Nat. Energy 3, 828 (2018).

[7] I. M. Peters, S. Sofia, J. Mailoa, and T. Buonassisi, RSC Adv. 6, 66911 (2016).

[8] S. Nishiwaki, S. Siebentritt, P. Walk, and M. Ch. Lux-Steiner, Prog. Photovoltaics Res. Appl. 11, 243 (2003).

[9] N. Gaillard, D. Prasher, M. Chong, A. Deangelis, K. Horsley, H. A. Ishii, J. P. Bradley, J. Varley, and T. Ogitsu, ACS Appl. Energy Mater. 2, 5515 (2019).

[10] S. H. Moon, S. J. Park, Y. J. Hwang, D.-K. Lee, Y. Cho, D.-W. Kim, and B. K. Min, Sci. Rep. 4, 4408 (2014). 
[11] B. Tell, J. L. Shay, and H. M. Kasper, Phys. Rev. B 4, 2463 (1971).

[12] H. Hiroi, Y. Iwata, H. Sugimoto, and A. Yamada, IEEE J. Photovoltaics 6, 1630 (2016).

[13] S. Merdes, R. Mainz, J. Klaer, A. Meeder, H. RodriguezAlvarez, H. W. Schock, M. C. Lux-Steiner, and R. Klenk, Sol. Energy Mater. Sol. Cells 95, 864 (2011).

[14] R. Kaigawa, A. Neisser, R. Klenk, and M. C. Lux-Steiner, Thin Solid Films 415, 266 (2002).

[15] A. Lomuscio, T. Rödel, T. Schwarz, B. Gault, M. Melchiorre, D. Raabe, and S. Siebentritt, Phys. Rev. Appl. 11, 054052 (2019).

[16] H. Azimi, T. Heumüller, A. Gerl, G. Matt, P. Kubis, M. Distaso, R. Ahmad, T. Akdas, M. Richter, W. Peukert, and C. J. Brabec, Adv. Energy Mater. 3, 1589 (2013).

[17] S. Fiechter, Y. Tomm, M. Kanis, R. Scheer, and W. Kautek, Phys. Status Solidi B 245, 1761 (2008).

[18] T. Schwarz, A. Lomuscio, S. Siebentritt, and B. Gault, Nano Energy 76, 105081 (2020).

[19] A. Lomuscio, M. Sood, M. Melchiorre, and S. Siebentritt, Phys. Rev. B 101, 085119 (2020).

[20] S. Merdes, R. Sáez-Araoz, A. Ennaoui, J. Klaer, M. C. LuxSteiner, and R. Klenk, Appl. Phys. Lett. 95, 213502 (2009).

[21] L. Weinhardt, O. Fuchs, D. Groß, G. Storch, E. Umbach, N. G. Dhere, A. A. Kadam, S. S. Kulkarni, and C. Heske, Appl. Phys. Lett. 86, 062109 (2005).

[22] M. Sood, A. Lomuscio, F. Werner, A. Nikolaeva, P. J. Dale, M. Melchiorre, J. Guillot, D. Abou-Ras, and S. Siebentritt, Solar RRL 5, 2100078 (2021).

[23] S. Merdes, D. Abou-Ras, R. Mainz, R. Klenk, M. C. LuxSteiner, A. Meeder, H. W. Schock, and J. Klaer, Prog. Photovoltaics Res. Appl. 21, 88 (2013).

[24] R. Klenk, J. Klaer, R. Scheer, M. C. Lux-Steiner, I. Luck, N. Meyer, and U. Rühle, Thin Solid Films 480-481, 509 (2005).

[25] H. Hiroi, Y. Iwata, S. Adachi, H. Sugimoto, and A. Yamada, IEEE J. Photovoltaics 6, 760 (2016).

[26] J. J. M. Binsma, L. J. Giling, and J. Bloem, J. Lumin. 27, 55 (1982).

[27] S. Shirakata, K. Saiki, and S. Isomura, J. Appl. Phys. 68, 291 (1990).

[28] J. R. Botha, M. S. Branch, P. R. Berndt, A. W. R. Leitch, and J. Weber, Thin Solid Films 515, 6246 (2007).

[29] G. Massé, J. Appl. Phys. 58, 930 (1985).

[30] T. Unold and L. Gütay, Adv. Charact. Tech. Thin Film Sol. Cells 1, 275 (2016).

[31] T. H. Gfroerer, Encyclopedia of Analytical Chemistry, edited by R. A. Meyers (John Wiley \& Sons Ltd, Chichester, 2000), pp. 9209-9231.

[32] C. Spindler, F. Babbe, M. H. Wolter, F. Ehré, K. Santhosh, P. Hilgert, F. Werner, and S. Siebentritt, Phys. Rev. Mater. 3, 090302 (2019).

[33] P. Wurfel, J. Phys. C: Solid State Phys. 15, 3967 (1982).

[34] P. Wurfel, Physics of Solar Cells: From Basic Principles to Advanced Concepts (WILEY-VCH Verlag GmbH \& Co. KGaA, Weinheim, 2009).

[35] F. Babbe, L. Choubrac, and S. Siebentritt, Appl. Phys. Lett. 109, 082105 (2016).

[36] T. Kirchartz, J. A. Márquez, M. Stolterfoht, and T. Unold, Adv. Energy Mater. 10, 1904134 (2020).
[37] See Supplemental Material at http://link.aps.org/supplemental/ 10.1103/PhysRevMaterials.5.055403 for more optical and electrical characterization results.

[38] C. Kittel, Introduction to Solid State Physics (John Wiley \& Sons, Inc., New Jersey, 2005).

[39] A. Thomere, C. Guillot-Deudon, M. T. Caldes, R. Bodeux, N. Barreau, S. Jobic, and A. Lafond, Thin Solid Films 665, 46 (2018).

[40] W. H. Koschel and M. Bettini, Phys. Status Solidi B 72, 729 (1975).

[41] A. Neisser, I. Hengel, R. Klenk, T. W. Matthes, J. ÁlvarezGarcía, A. Pérez-Rodríguez, A. Romano-Rodríguez, and M. C. Lux-Steiner, Sol. Energy Mater. Sol. Cells 67, 97 (2001).

[42] N. Guijarro, M. S. Prévot, M. Johnson, X. Yu, W. S. Bourée, X. A. Jeanbourquin, P. Bornoz, F. Le Formal, and K. Sivula, J. Phys. D: Appl. Phys. 50, 044003 (2016).

[43] J. I. Pankove, Optical Processes in Semiconductors (Dover, New York, 1971).

[44] T. Schmidt, K. Lischka, and W. Zulehner, Phys. Rev. B 45, 8989 (1992).

[45] C. Spindler, T. Galvani, L. Wirtz, G. Rey, and S. Siebentritt, J. Appl. Phys. 126, 175703 (2019).

[46] D. S. Max Hilaire Wolter, A. Lomuscio, T. P. Weiss, P. Thiele, P. J. Dale, and S. Siebentritt (unpublished).

[47] M. H. Wolter, Doctoral thesis, University of Luxembourg, 2019, http://hdl.handle.net/10993/39611.

[48] S. Siebentritt, N. Papathanasiou, and M. C. Lux-Steiner, Phys. B (Amsterdam, Neth.) 376-377, 831 (2006).

[49] S. Siebentritt, N. Rega, A. Zajogin, and M. C. Lux-Steiner, Phys. Status Solidi C 1, 2304 (2004).

[50] J. Krustok, H. Collan, M. Yakushev, and K. Hjelt, Phys. Scr. T79, 179 (1999).

[51] G. Rey, G. Larramona, S. Bourdais, C. Choné, B. Delatouche, A. Jacob, G. Dennler, and S. Siebentritt, Sol. Energy Mater. Sol. Cells 179, 142 (2018).

[52] K. F. Tai, T. Gershon, O. Gunawan, and C. H. A. Huan, J. Appl. Phys. 117, 235701 (2015).

[53] I. Dirnstorfer, M. Wagner, D. M. Hofmann, M. D. Lampert, F. Karg, and B. K. Meyer, Phys. Status Solidi A 168, 163 (1998).

[54] A. Bauknecht, S. Siebentritt, J. Albert, and M. C. Lux-Steiner, J. Appl. Phys. 89, 4391 (2001).

[55] K. Kazlauskas, G. Tamulaitis, A. Žukauskas, M. A. Khan, J. W. Yang, J. Zhang, G. Simin, M. S. Shur, and R. Gaska, Appl. Phys. Lett. 83, 3722 (2003).

[56] A. D. Wright, R. L. Milot, G. E. Eperon, H. J. Snaith, M. B. Johnston, and L. M. Herz, Adv. Funct. Mater. 27, 1700860 (2017).

[57] J. K. Larsen, K. Burger, L. Gütay, and S. Siebentritt, in 2011 37th IEEE Photovoltaic Specialists Conference (IEEE, Seattle, WA, 2011), pp. 000396-000401.

[58] B. I. Shklovskii and A. L. Efros, Electronic Properties of Doped Semiconductors (Springer-Verlag, Berlin, Heidelberg, 1984).

[59] A. P. Levanyuk and V. V. Osipov, Phys. Usp. 24, 187 (1981).

[60] P. Blood and J. W. Orton, The Electrical Characterization of Semiconductors: Majority Carriers, and Electron States (Academic, London, 1992).

[61] F. Werner and S. Siebentritt, Phys. Rev. Appl. 9, 054047 (2018).

[62] T. P. Weiss, A. Redinger, D. Regesch, M. Mousel, and S. Siebentritt, IEEE J. Photovoltaics 4, 1665 (2014). 
[63] T. Walter, D. Braunger, H. Dittrich, C. Köble, R. Herberholz, and H. W. Schock, Sol. Energy Mater. Sol. Cells 41, 355 (1996).

[64] R. Scheer, M. Alt, I. Luck, and H. J. Lewerenz, Sol. Energy Mater. Sol. Cells 49, 423 (1997).

[65] J. H. Schön and E. Bucher, Phys. Status Solidi A 171, 511 (1999).

[66] R. Klenk, U. Blieske, V. Dieterle, K. Ellmer, S. Fiechter, I. Hengel, A. Jäger-Waldau, T. Kampschulte, C. Kaufmann, J. Klaer, M. C. Lux-Steiner, D. Braunger, D. Hariskos, M. Ruckh, and H. W. Schock, Sol. Energy Mater. Sol. Cells 49, 349 (1997).

[67] K. Siemer, J. Klaer, I. Luck, J. Bruns, R. Klenk, and D. Bräunig, Sol. Energy Mater. Sol. Cells 67, 159 (2001).

[68] N. Barange, V. B. Chu, M. Nam, I.-H. Ahn, Y. D. Kim, I. K. Han, B. K. Min, and D.-H. Ko, Adv. Energy Mater. 6, 1601114 (2016).
[69] A. Kotbi, B. Hartiti, S. Fadili, A. Ridah, and P. Thevenin, Appl. Phys. A 123, 379 (2017).

[70] T. Ohashi, Y. Hashimoto, and K. Ito, Jpn. J. Appl. Phys. 38, L748 (1999).

[71] R. Mainz, F. Streicher, D. Abou-Ras, S. Sadewasser, R. Klenk, and M. C. Lux-Steiner, Phys. Status Solidi A 206, 1017 (2009).

[72] G. He, C. Yan, J. Li, X. Yuan, K. Sun, J. Huang, H. Sun, M. He, Y. Zhang, J. A. Stride, M. A. Green, and X. Hao, ACS Appl. Energy Mater. 3, 11974 (2020).

[73] S. Kim, T. Nagai, H. Tampo, S. Ishizuka, and H. Shibata, Prog. Photovoltaics Res. Appl. 28, 816 (2020).

[74] F. Larsson, N. S. Nilsson, J. Keller, C. Frisk, V. Kosyak, M. Edoff, and T. Törndahl, Prog. Photovoltaics Res. Appl. 25, 755 (2017).

[75] B. Marsen, H. Wilhelm, L. Steinkopf, S. Klemz, T. Unold, R. Scheer, and H. W. Schock, Thin Solid Films 519, 7224 (2011). 